\title{
Differentials in poisoning rates of young Australian children according to residential location and geographical remoteness
}

\section{P J O'Connor}

Injury Prevention 2005;11:204-206. doi: 10.1136/ip.2004.005926

\begin{abstract}
Objectives: To assess differentials in the poisoning rates of children aged $0-4$ years according to residential location and geographical remoteness.

Design: Cross sectional study based on hospitalizations.

Setting: Australia.

Subjects: Children aged $0-4$ years admitted to hospital due to poisoning during the financial year 1996-97.

Main outcome measures: Crude rates of hospitalization.

Results: The rate of hospitalization due to poisoning peaked in the third year and second year of life for medicinal and non-medicinal substances respectively. Rates were significantly higher among children aged $0-4$ years residing in rural and remote areas when compared with those residing in metropolitan areas, and rate differentials increased with geographical remoteness.

Conclusions: The observed differentials suggest the need for targeted research and prevention efforts aimed at rural and remote area communities. A detailed empirical study is recommended, involving the assessment of risk factors and an in-home hazard checklist, as a precursor to any intervention program.
\end{abstract}

Correspondence to: Dr P J O'Connor; peteroconnor@netspace. net.au

Accepted 3 March 2005 t thas been estimated that the cost of poisoning of children aged less than 5 years (preschoolers) is more than $\mathrm{A} \$ 20$ million per year in Australia. ${ }^{1}$ Poisoning in this age group is the third most common reason for presentation at a hospital emergency department, after falls and impact with an object. ${ }^{2}$ Hospital admission data show that poisoning accounted for $13.5 \%$ of total reported injury in preschoolers and was the third highest reported injury category after falls and other unintentional injuries in this group. ${ }^{3}$ In consideration of this, poisoning among preschoolers was chosen as one of four priority areas of the National Injury Prevention Plan. ${ }^{3}$

In order to provide background information about the problem, two national statistical reports were prepared, ${ }^{45}$ the full details of which are available from the website of the Australian Institute of Health and Welfare (AIHW) National Injury Surveillance Unit (http://www.nisu.flinders.edu.au). These reports raised a number of issues of potential wider interest. Notable among these was a strong differential in poisoning rates for preschoolers according to residential location-findings that had not been reported before in the literature for other countries. These differentials are reported in this article for an international audience as they may have implications for research and the targeting of prevention efforts beyond Australia.

\section{METHODS}

\section{Data sources}

Hospital separations data for the financial year 1996-97 made available by the AIHW. The AIHW database includes data from public acute and Department of Veterans' Affairs hospitals, and private and psychiatric hospitals.

\section{Case selection criteria}

The case selection criteria were: (1) an age of less than 5 years and (2) admitted to a hospital with a principal diagnosis of injury arising from an external cause coded in the range E850-858 ("Accidental poisoning by drugs, medicaments and biologicals"; referred to as medicinal substances or medicinals) and E860-869 ("Accidental poisoning by other solid and liquid substances, gases, and vapours"; referred to as non-medicinal substances or nonmedicinals). ${ }^{6}$ As a single poisoning can lead to multiple hospital admissions, all readmissions were excluded in order to provide incidence estimates focusing on the number of persons hospitalized rather than the number of admissions.

\section{Rate calculation and confidence intervals}

Population based rates were produced using unpublished population estimates for 1996-97 provided by the Australian Bureau of Statistics at the level of statistical local area.

Rates of hospitalization were produced according to the Rural, Remote, and Metropolitan Area classification system (RRMA), ${ }^{7}$ involving aggregation of statistical local areas, in order to study the geographical distribution of poisoning by place of residence.

Where case numbers are small, the effect of chance variation on rates can be large. Therefore, 95\% confidence intervals were placed around rates as a guide to the size of this variation, based on a Poisson assumption about the number of cases in a time period. Chance variation alone would be expected to lead to a rate outside the interval only once out of 20 occasions.

\section{RESULTS}

The crude rate of medicinal poisoning hospitalizations in 0-4 year olds (196/100 000 population) was more than double the crude rate of non-medicinal poisoning in this age group (79/100 000 population) in the financial year 1996-97.

The rates peaked in the third year of life for medicinal substances and the second year for non-medicinal substances (fig 1). Fifty four percent of the preschooler medicinal poisoning cases admitted to hospital were male, compared with 58\% for non-medicinal poisoning cases.

Of the hospitalizations for which a place of poisoning was noted $(80 \%$ of medicinal poisonings and $75 \%$ of

Abbreviations: AlHW, Australian Institute of Health and Welfare. 


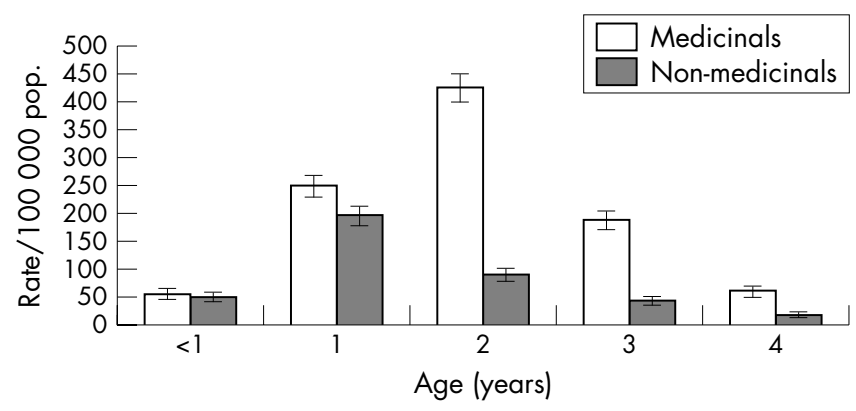

Figure 1 Estimated new incident cases of poisoning from medicinal and non-medicinal substances in children aged $0-4$ years, Australia 1996-97 financial year (age specific rate based on hospital separations data)

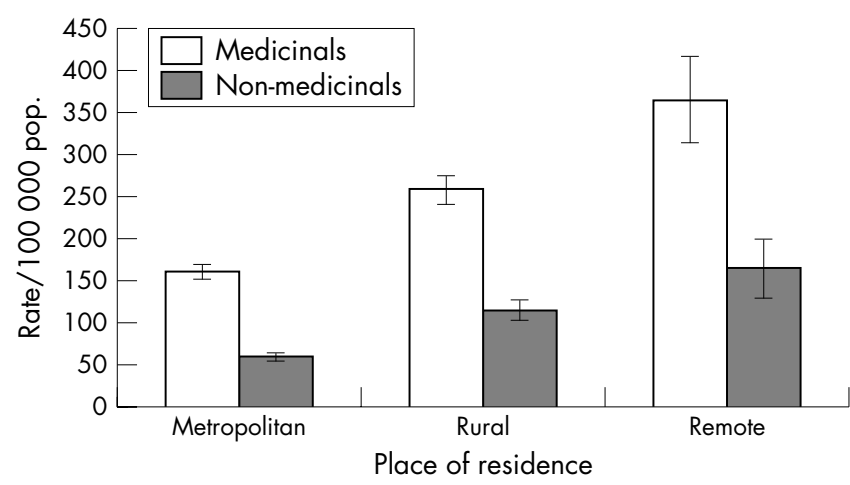

Figure 2 Estimated new incident cases of poisoning from medicinal and non-medicinal substances in children aged $0-4$ years by place of residence, Australia 1996-97 financial year (crude rate based on hospital separations data)

non-medicinal poisonings), nearly all occurred in the home (96\% and 91\% for medicinal and non-medicinal substances respectively).

Rates of hospitalization for both medicinal and nonmedicinal poisonings were significantly higher among preschoolers resident in rural and remote areas compared with metropolitan areas and rate differentials increased with geographical remoteness (see fig 2).

\section{DISCUSSION}

This study showed that nearly three in every 1000 children aged 0-4 years in the financial year 1996-97 were hospitalized due to poisoning in that year and the hospitalization rate was highest for medicinal poisonings.

Most of the poisonings occurred in the home. Due to the limitations of ICD9 coding it was not possible to determine whether this was the child's own home or someone else's home. Assessment of this issue elsewhere in Australia suggested that in most cases it would have occurred in the child's own home. ${ }^{8}$ It was therefore relevant to consider differentials in rates of poisoning according to residential location of the poisoned child.

Children living in rural and remote areas of Australia had higher crude rates of poisoning than children living in metropolitan areas and the rate differentials increased with geographical remoteness. Unfortunately, it was not possible to determine whether these differentials reflected differences in the age distributions of the areas by individual year of age, as this information was not available. The reported rate differentials could also reflect differences in hospital admission policies between metropolitan, rural, and remote area hospitals, as suggested by Hockey et al. ${ }^{2}$ However, unless convincing evidence is provided to support this suggestion,

\section{Key points}

- Children living in rural and remote areas of Australia had higher crude rates of poisoning than children living in metropolitan areas and the rate differentials increased with geographical remoteness.

- The causes and prevention of this problem have received little attention in the literature.

- A case control study has been planned, involving an assessment of the home environment and risk factors of young children residing in rural and remote areas.

- This will be implemented through regional child health clinics that undertake periodic health checks and home visits during the early childhood period.

the noted rate differentials should not be ignored. Contrasts in all-ages poisoning death rates by area of residence ${ }^{9}{ }^{10}$ add weight to the suggestion that specific targeting of rural and remote area communities is required.

Unfortunately, little is currently known about the special measures required to prevent poisoning of young children in rural and remote areas as the topic has not been researched before. Even in Australia, where poisoning among preschoolers was chosen as one of four priority areas of the National Injury Prevention Plan $^{3}$ and the differentials reported here have been known since the year 2000, the topic has not received further attention. Until there is a better understanding of the causal factors, prevention requirements cannot be specified. Indeed, at present any discussion of prevention will necessarily be limited to putative solutions based on experience gained in metropolitan environments because this is the site of the available published research and it may not be applicable in rural and remote areas.

Considering the lack of an evidence base for the causes and prevention of the problem it is recommended that a research study be undertaken involving an assessment of the home environment and risk factors of young children residing in rural and remote areas, including farms, which could possibly be implemented through regional child health clinics. As a component of the research an inventory of poisoning hazards should be constructed. None of the available Australian checklists (see http://www.agsafe.com.au and http://www. farmsafe.org.au) is totally adequate for the present purpose and these need to be extended.

It is not known whether the geographical differentials reported for Australia also occur in other countries as this information has not been reported before in the international literature. It is unlikely that the differentials are unique to Australia. Chemical hazards and exposures are likely to be greater in the rural and agricultural areas of most countries as poisons are used extensively and intensively to control pests and crop and animal diseases. In countries that have sizeable rural populations, differentials in childhood poisoning rates and exposures should be examined as there is the potential for substantially increased rates and the need for research into effective control measures.

\section{CONCLUSIONS}

There are strong differentials in the poisoning rates of young Australian children according to residential location and geographical remoteness. These differentials should be examined in other countries and reported in the literature. In addition, research into the risk factors, causes, and prevention is required in order to provide the much needed evidence base for prevention efforts. 


\section{ACKNOWLEDGEMENTS}

Nina O'Connor provided assistance in the preparation of the manuscript.

\section{REFERENCES}

1 Moller J. Estimated cost of injury by poisoning Australia 1995-96. Adelaide: Research Centre for Injury Studies, 1998.

2 Hockey R, Reith D, Miles E. Childhood poisoning and ingestion. Brisbane: QISU Injury Bulletin, Number 60, 2000.

3 Helps Y, Cripps R, Harrison J. Hospital separations due to injury and poisoning, Australia 1999-00. Adelaide: Injury Research and Statistics Series, Number 15, 2002.

4 O'Connor PJ. Accidental poisoning of preschool children from non-medicinal substances in Australia. Adelaide: Research Centre for Injury Studies, Flinders University of South Australia, 2000.
5 O'Connor PJ. Accidental poisoning of preschool children from medicinal substances, Australia. Adelaide: Research Centre for Injury Studies, Flinders University of South Australia, 2001.

6 World Health Organization. International classification of diseases, 9th revision, clinical modification, Australian version (ICD-9-CM). Geneva: WHO, 1995.

7 Arundell L. Rural, Remote and Metropolitan Zones Classification: A Classification of Australia as at 30 June 1986 and a Methodology for 1991 Census Data. Canberra: Department of Primary Industries and Energy, 1991

8 Routley V, Ozanne-Smith J, Ashby K. Poisonings in early childhood. Hazard No 20, Victorian Injury Surveillance System, Monash University Accident Research Unit, June, 1996.

9 Public Health Division. The Health of the People of New South WalesReport of the Chief Health Officer. Sydney: NSW Health Department, 1997:156.

10 Moller J. The spatial distribution of injury deaths in Australia: urban, rural and Remote Areas. Adelaide: AlHW National Injury Surveillance Unit, 1994. 